\title{
Migrasomes: a new organelle of migrating cells
}

\author{
Cell Research (2015) 25:1-2. doi:10.1038/cr.2014.146; published online 7 November 2014
}

Cell migration is a multi-step process that involves the coordinated action of signaling networks, cytoskeletal dynamics and vesicular trafficking, leading to protrusion and adhesion at the leading edge of cells and contraction and detachment at their rear. In a recent paper in Cell Research, Ma et al. describe the biogenesis of a new exosome-like organelle - named migrasomes that derive from retraction fibers at the rear of migrating cells and their potential roles in inter-cellular signaling.

Cell migration is a complex process of fundamental importance in numerous physiological or pathological processes, including tissue development, repair and growth, as well as during cancer cell metastasis [1-3]. Cells use multiple modes of migration depending on the properties of the extracellular matrix (ECM) upon which they move, for example its architecture (two or three dimensional), stiffness and composition, and also on cellular determinants, such as cell-cell and cell-ECM adhesiveness, traction forces and proteolysis [4]. However, in general cell migration is a cyclical process involving: 1) lamellipodial protrusion at the leading edge, 2) cell adhesion by integrin receptors, 3) creation of cell tension, 4) cell contraction, and 5) retraction of the cell rear end. Accomplishing these steps requires integration of signaling, cytoskeletal dynamics, adhesion and membrane trafficking [1-4].

Although most research on migration focuses on events at the cell's leading edge, the rear end (trailing edge) is equally important because without the coordinated disassembly of cell adhesions and the recycling of ECM receptors, cells would not move [5]. Long tubular cytoplasmic expansions called retraction fibers (fibrils) are observed at the rear end of forward migrating cells [6], and also during abrupt detachment from substrates [7]. At the tips of these thin extensions, membrane shedding can occur, leaving integrin-containing vesicles attached to the substrate along the migratory path $[7,8]$. Previously thought to be passively deposited cell remnants, Ma et al. [9] provide evidence for the active transport of materials from the cell body to a specialized subcompartment within these fibers, called migrasomes. As the cell moves and the fibers retract, the migrasomes are released to function as a potentially new and structurally distinct category of exosome-like vesicles that transmit signals between migrating cells (Figure 1).

Exosomes are micro-vesicles of endosomal origin thought to be released after fusion of multivesicular endosomes (MVBs) with the plasma membrane [10]. Exosomes, which contain cytosolic contents including miRNAs, as well as lipids and membrane receptors, are released from one cell and believed to bind and/or be taken up by another, providing a means of intercellular communication. Interestingly, exosomes have been shown to induce cell migration and invasion in cancer models [11]. Proteomic analysis of purified migrasomes suggests that they are compositionally related to exosomes [9], and in particular are enriched in tetraspanins, membrane protein markers of MVBs. However, unlike exosomes, the released migrasomes are large in diameter $(0.5-1.2 \mu \mathrm{m})$ and contain variable numbers of small internal vesicles.

To gain more insights into migrasome biogenesis, Ma et al. expressed and tracked GFP-tagged tetraspanin-4 (TSPAN4-GFP), a protein previously localized to retraction fibers [7] and shown to be enriched in isolated migrasomes. By time-lapse microscopy of migrating cells [9], migrasomes form as bulb-like structures at the tips of, or at the intersections between, retraction fibers. Importantly, these bulbs continue to grow in diameter, receiving cytosolic input continuously over an $\sim 2 \mathrm{~h}$ period as the migrating cell advances. When the retraction fibers break, migrasomes are released as a package of vesicles enclosed within a single limiting membrane. Eventually the migrasome dissociates from the substrate and is released into the medium, unless it is engulfed by an oncoming cell (see below).

Not surprisingly, given their relationship with retraction fibers, migrasome formation is dependent on cell migration. Thus, the number of migrasomes formed per cell is increased when migration is enhanced (e.g., by coating surfaces with fibronectin, which stimulates cell adhesion and migration [12] or by knocking down SHARPIN, an endogenous inhibitor of $\beta 1$ integrin activation [13]) and decreased when migration is inhibited (e.g., by treating cells with blebbistatin, a myosin II blocker [14]). Importantly, given cell type differences in migration and differences in migration on $2 \mathrm{D}$ vs $3 \mathrm{D}$ matrices and in vivo, the authors used TSPAN4-GFP transfection and scanning electron microscopy to identify migrasomes formed by multiple cell 


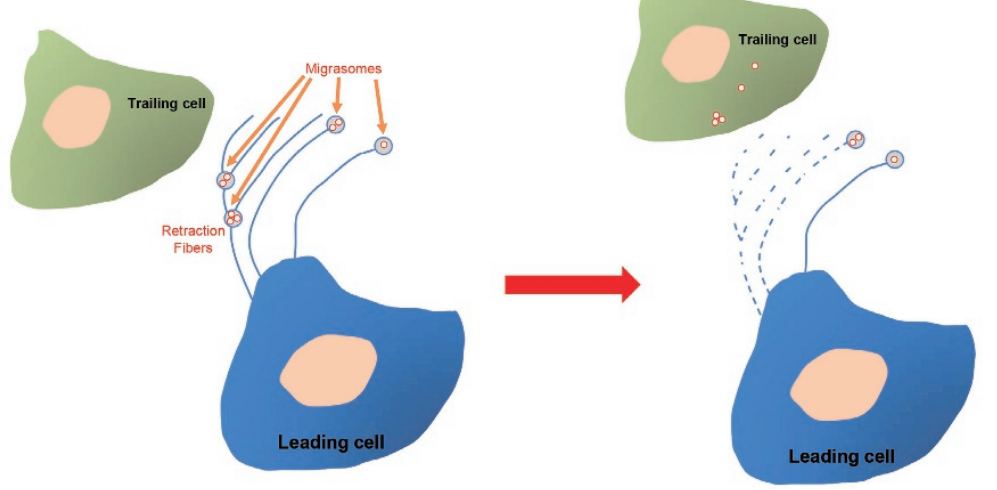

Figure 1 Migrasomes form within retraction fibers emerging from the trailing edge of migrating cells. They are released and deposited on the substrate as the fibers collapse. Migrasomes may represent a vector for inter-cellular communication as they can be engulfed by trailing cells, providing guidance cues or other information.

lines and under multiple conditions. They also identified externally deposited MVBs reminiscent of migrasomes by transmission electron microscopy in various mouse tissues, providing strong evidence for migrasome deposition under physiologically relevant conditions. Although morphologically reminiscent of MVBs, electron micrographs of migrasomes growing within retraction fibers show a collection of free vesicles and cytosolic content and not the deposition of intact MVBs. When the retraction fiber breaks, the released migrasome is surrounded by a single membrane, presumably derived from the surface membrane of the retraction fiber. This is important because the outer membrane of an MVB, unlike the retraction fiber membrane, would be topologically reversed exposing the cytoplasmic surface to the extracellular media.

Migrasomes represent a distinct type of extracellular vesicle deposited through a unique mechanism and left in the tracks of migrating cells. Clearly, further work is necessary both to define the cellular components and potential signaling molecules that accumulate in growing bulbs along retraction fibers for release in migrasomes, and to identify the intracellular targeting signals and mechanisms that deposit them there. Most important is to understand the function of migrasomes. One clue comes from striking time-lapse videos showing that other cells following in the path of their depositors can take up migrasomes [9]. Which signals are activated upon contact with and/or internalization of deposited migrasomes? What inter-cellular messages might they be delivering? Could they serve as migratory benchmarks or guidance cues? The discovery of these extracellular vesicles raises many new questions and opens many avenues for future research.

\section{Bruno da Rocha-Azevedo ${ }^{1,2}$, Sandra L Schmid ${ }^{1}$}

${ }^{1}$ Department of Cell Biology, University of Texas Southwestern Medical Center, Dallas, Texas, USA; ${ }^{2}$ Federal University of Rio de Janeiro, Rio de Janeiro, Brazil

Correspondence: Sandra L Schmid

E-mail: sandra.schmid@utsouthwestern.edu

\section{References}

1 Ridley AJ, Schwartz MA, Burridge K, et al. Science 2003; 302:1704-1709.

2 Ulrich F, Heisenberg CP. Traffic 2009; 10:811-818.

3 Lauffenburger DA, Horwitz AF. Cell 1996; 84:359-369.

4 Friedl P, Wolf K. J Cell Biol 2010; 188:1119.

5 Webb DJ, Parsons JT, Horwitz AF. Nat Cell Biol 2002; 4:E97-E100.

6 Palecek SP, Schmidt CE, Lauffenburger DA, et al. J Cell Sci 1996; 109(Pt 5):941952.

7 Yamada M, Mugnai G, Serada S, et al. Cell Adh Migr 2013; 7:304-314.

8 Mayer C, Maaser K, Daryab N, et al. Eur J Cell Biol 2004; 83:709-715.

9 Ma L, Ying L, Peng J, et al. Cell Res 2015; 25:24-38.

10 Février B, Raposo G. Curr Opin Cell Biol 2004; 16:415-421.

11 Luga V, Zhang L, Viloria-Petit AM, et al. Cell 2012; 151:1542-1556.

12 Pankov R, Yamada KM. J Cell Sci 2002; 115:3861-3863.

13 Rantala JK, Pouwels J, Pellinen T, et al. Nat Cell Biol 2011; 13:1315-1324.

14 Kovacs M, Toth J, Hetenyi C, et al. J Biol Chem 2004; 279:35557-35563. 